\title{
The performance of the ATLAS Inner Detector Trigger Algorithms in pp collisions at the LHC
}

\author{
Mark Sutton
University of Sheffield
ATLAS Collaboration
}

9th June 2010 
X11 Topical Seminar IPRD, Siena - 7-10 th June 2010

\section{Preface}

- LHC took it first $900 \mathrm{GeV}$ collisions in November 2009

- $\mathrm{L}=\sim 9 \mu \mathrm{b}^{-1}$ at $\sim 5 \times 10^{26} \mathrm{~cm}^{-2} \mathrm{~s}^{-1}$

- Since March 2010, $7 \mathrm{TeV}$ collisions

- $\mathrm{L} \sim 15 \mathrm{nb}^{-1}$ at $\sim 2 \times 10^{29} \mathrm{~cm}^{-2} \mathrm{~s}^{-1}$

- Bunch crossing every $25 \mathrm{~ns}-40 \mathrm{MHz}$ rate

- Data storage capability $\sim 200 \mathrm{~Hz}$

- Reduction of around 200000 to 1 required

- Eventually expect up to 25 (soft) $p p$ interactions per bunch crossing

- Interesting high $p_{T}$ interactions complicated by "pile-up"

- Essentially all interesting signatures contain leptons or high momentum tracks

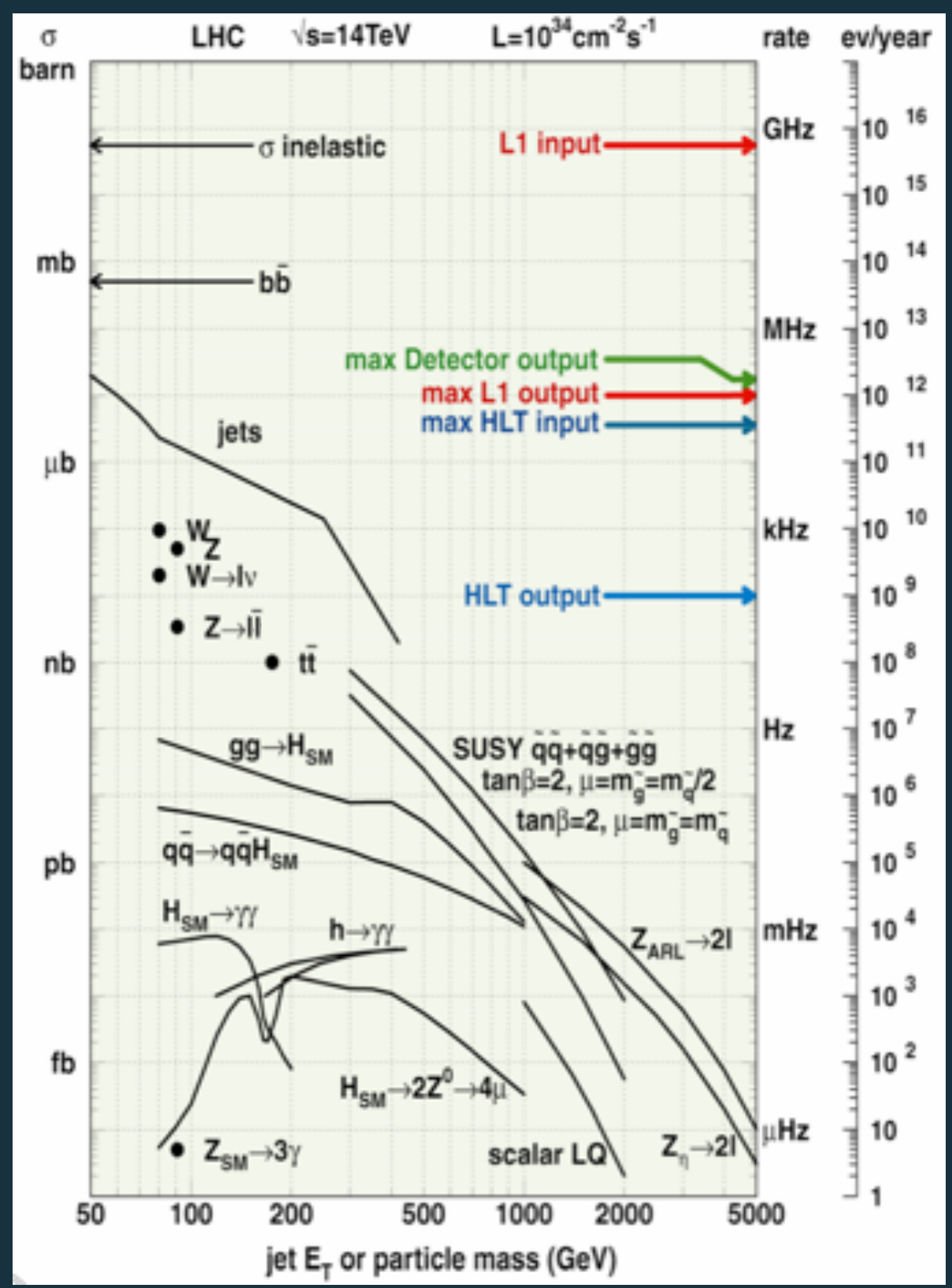

M.Sutton - The performance of the ATLAS Inner Detector Trigger 


\section{The ATLAS Detector}

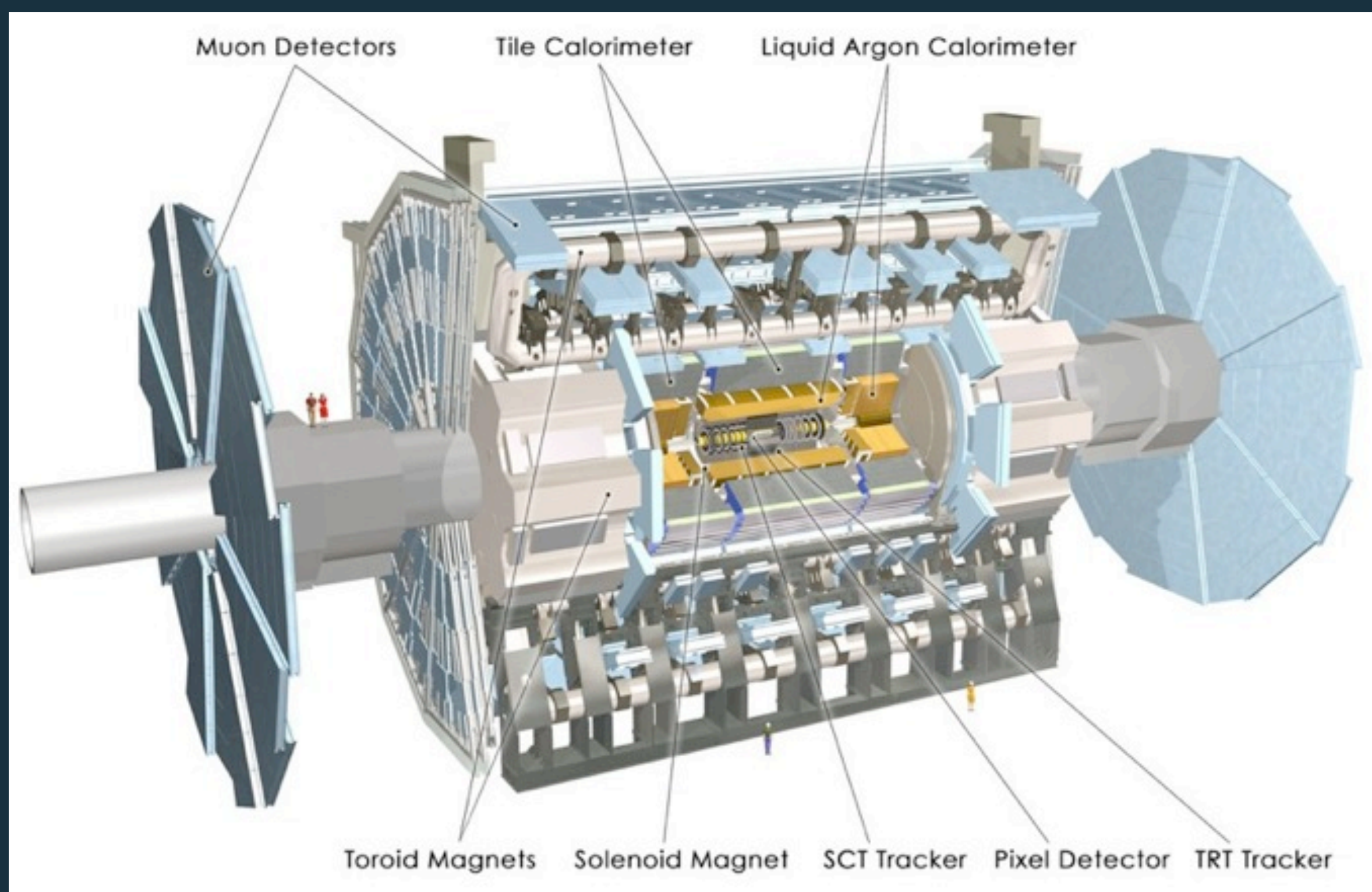

M.Sutton - The performance of the ATLAS Inner Detector Trigger 
X11 Topical Seminar IPRD, Siena - 7-10 th June 2010

\section{The Inner Detector}

- Transition Radiation Tracker (TRT)

- Radius $2 \mathrm{~mm}$ straw tubes

- Semiconductor Tracker (SCT)

- $80 \mu \mathrm{m}$ pitch Silicon strip detectors, double layered, $40 \mathrm{mrad}$ stereo

- Pixel Detector

- Silicon pixel detector $50 \times 400 \mu$ m pixels

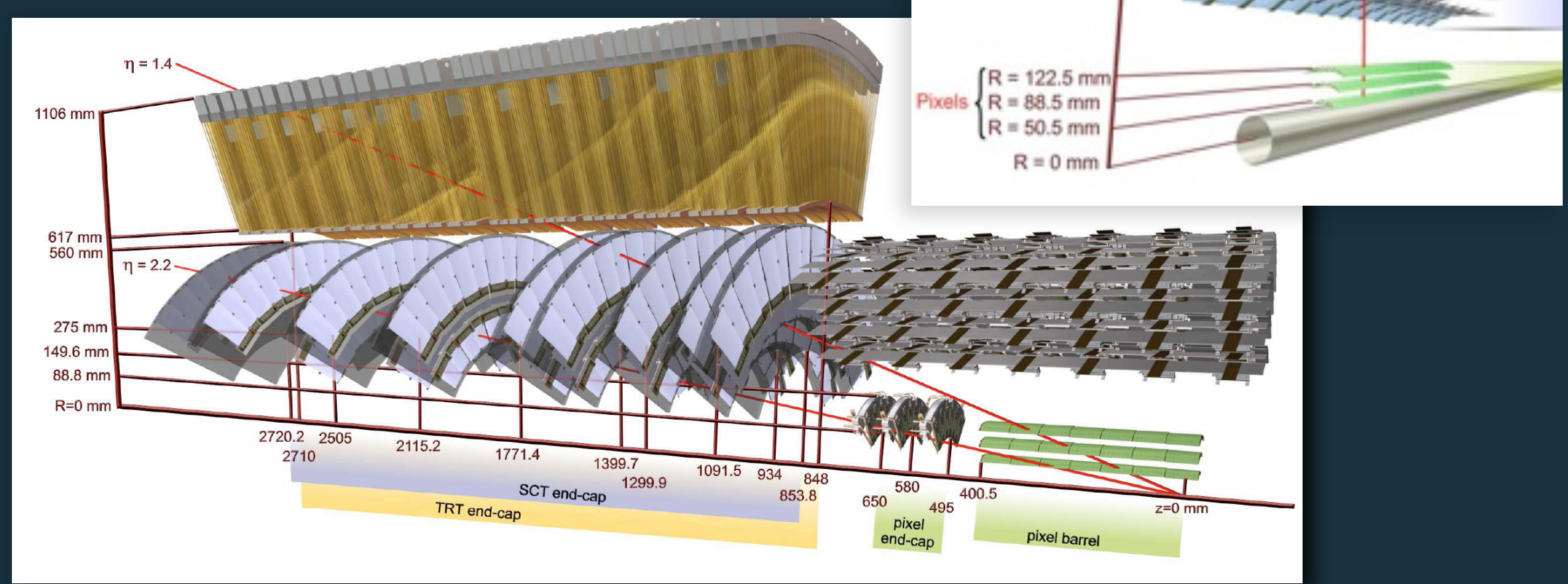




\section{The ATLAS Semiconductor Tracker}

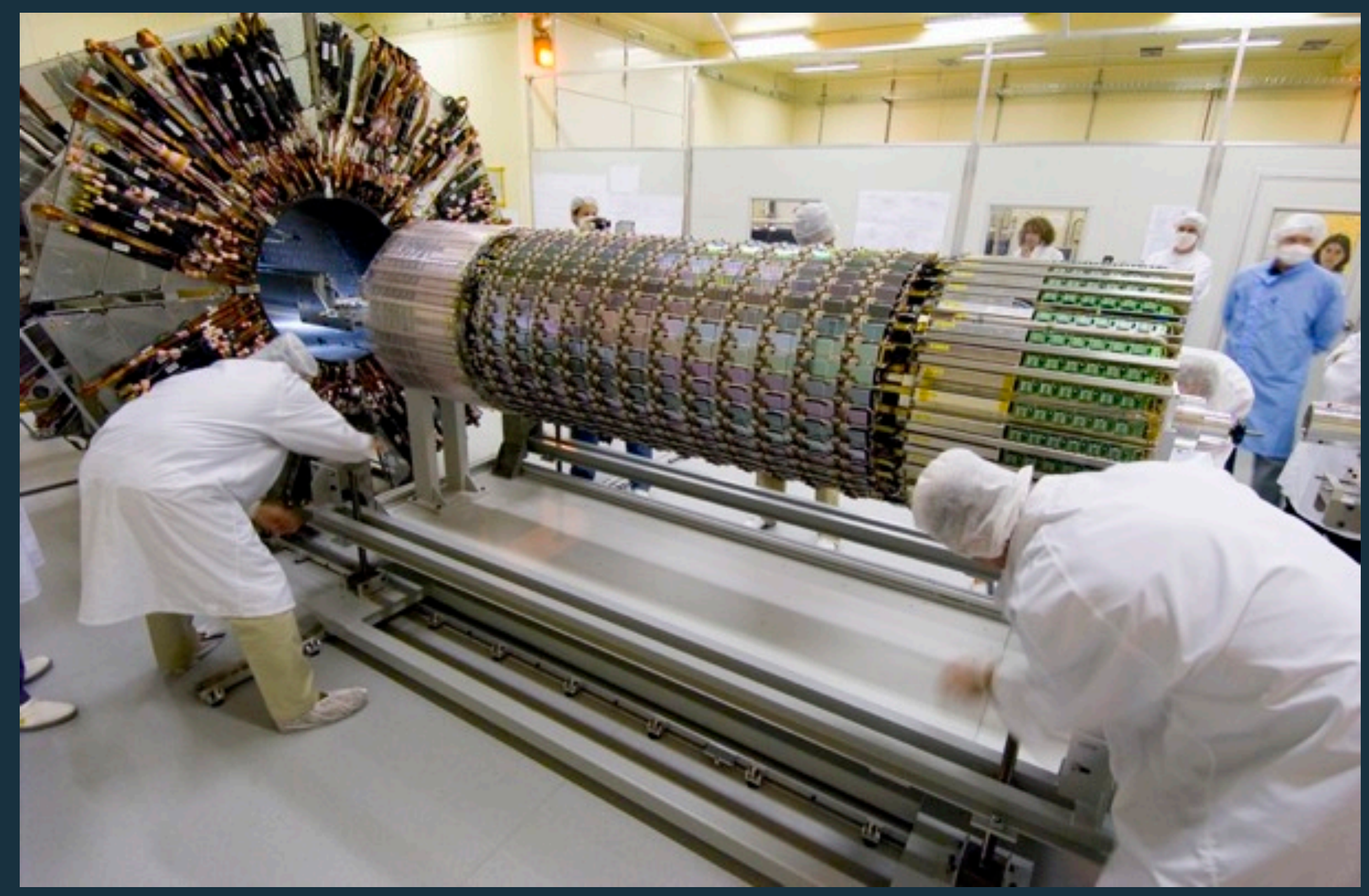

M.Sutton - The performance of the ATLAS Inner Detector Trigger 


\section{The ATLAS Trigger System}

- Level 1

- Hardware based, pipelined trigger, coarse granularity, largely Calorimeter and Muon system based.

- 2.5 microsecond latency

- High Level Trigger - Level 2 and Event Filter

- Software based, farms of commodity CPU's and ethernet

- Level 2

- Seeded by Level 1 in Regions of Interest

- Full detector granularity, all detector subsystems

- Event Filter

- Seeded by Level 2

- Access to complete event, reconstruction similar to Offline

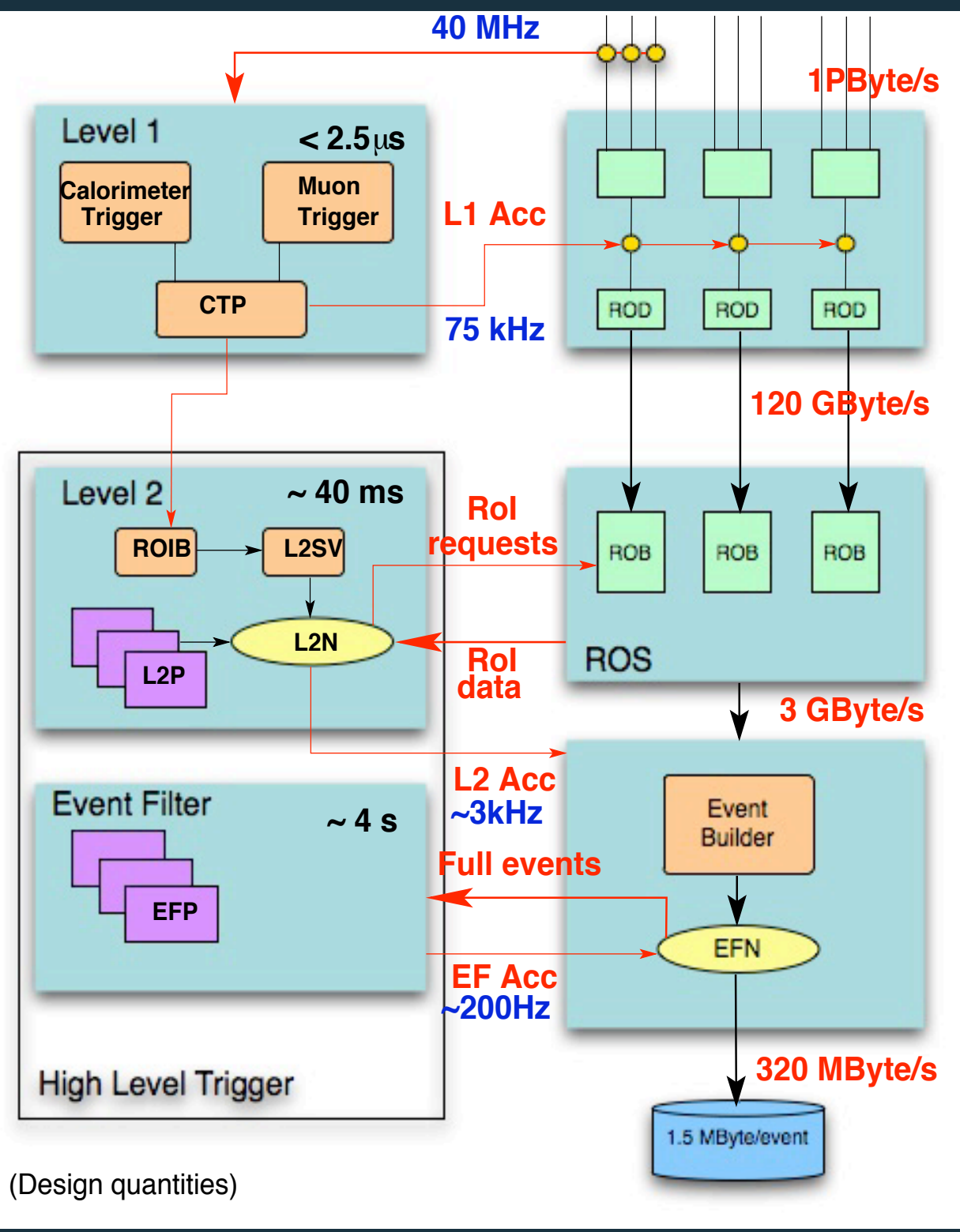

M.Sutton - The performance of the ATLAS Inner Detector Trigger 


\section{Collected data at $7 \mathrm{TeV}$}

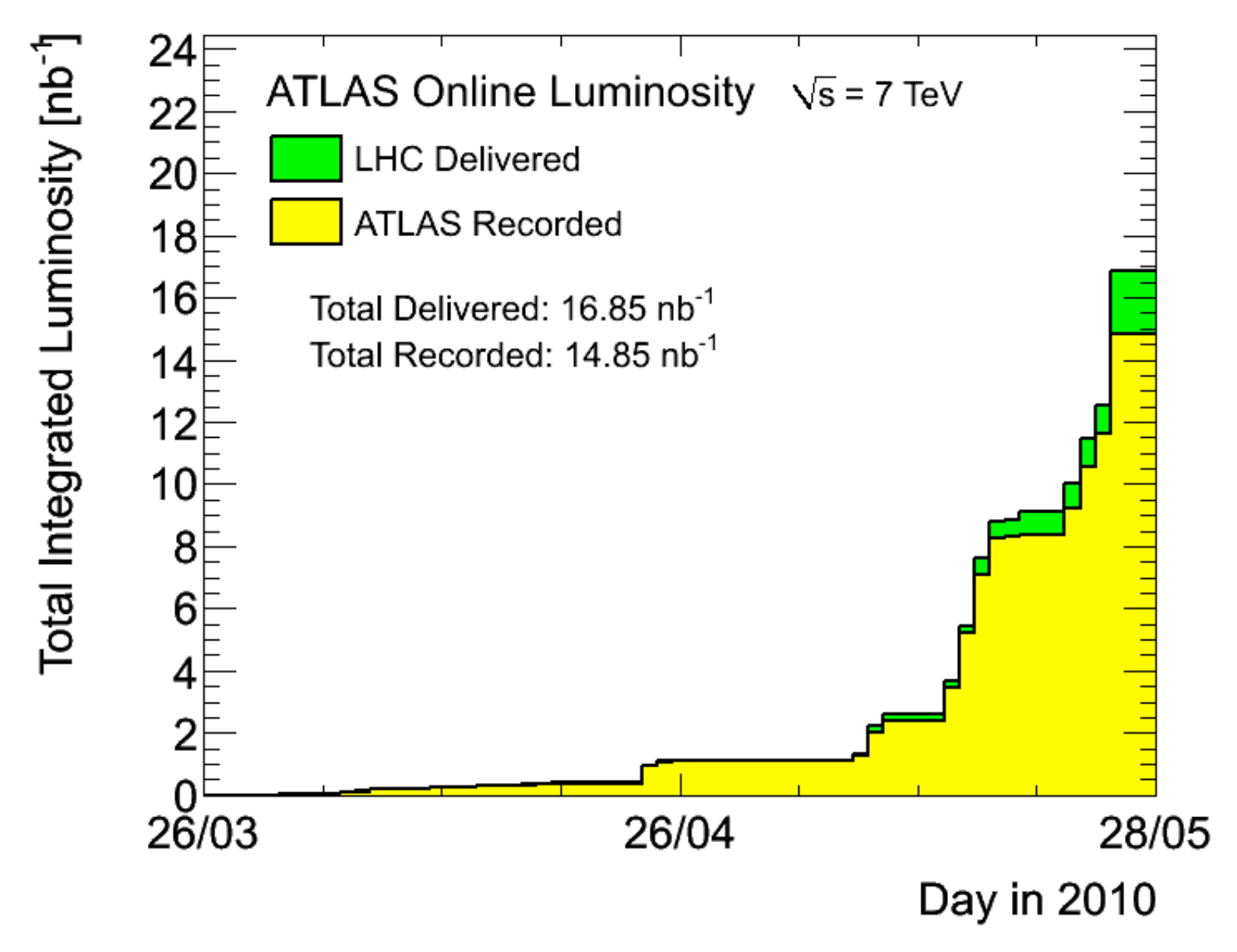

- LHC provided the first collisions at $7 \mathrm{TeV}$ at the end of March 2010 - So far, over 800 million events written to tape with stable beams 


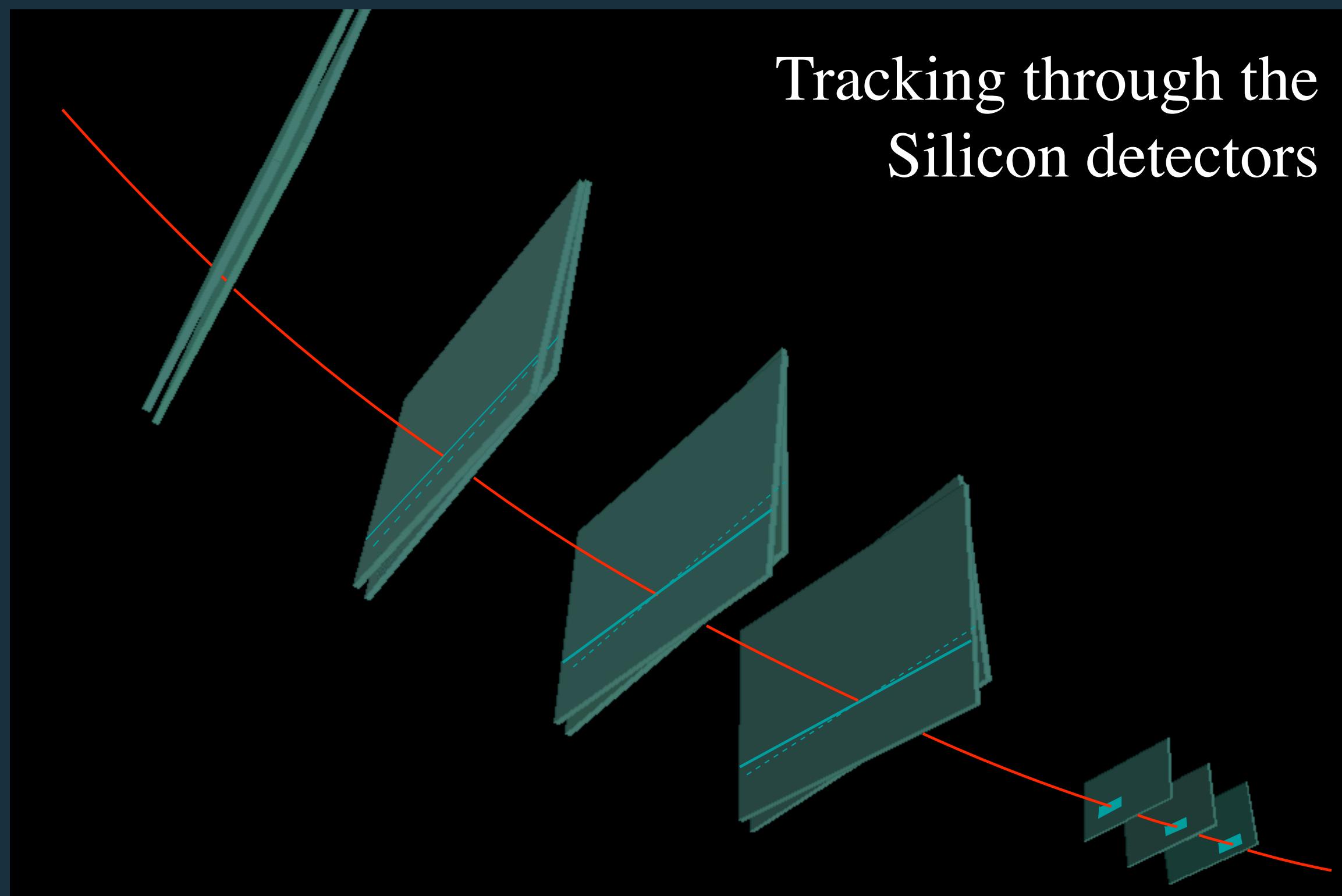




\section{Track Selection}

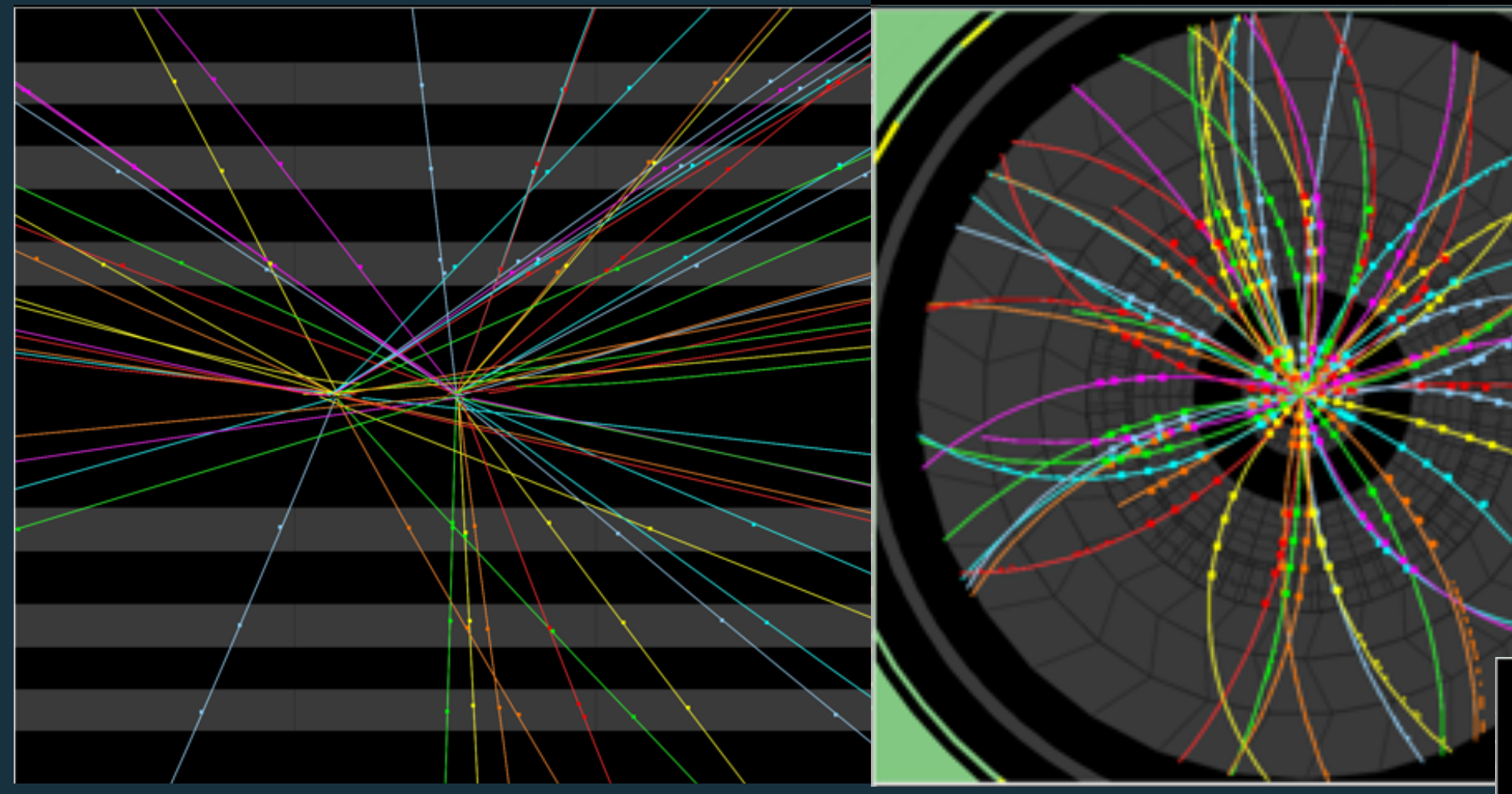

- Good Offline tracks were selected from $900 \mathrm{GeV}$ or $7 \mathrm{TeV}$ collisions

- $|\eta|<2.5, \mathrm{p}_{\mathrm{T}}>1 \mathrm{GeV}$ (no $\mathrm{p}_{\mathrm{T}}$ cut for distributions versus $\mathrm{p}_{\mathrm{T}}$ )

- Number of pixel hits $>0$, Number of SCT clusters $>5$

- consistent with Offline vertex

- The performance of the HLT Level 2 and EventFilter tracking was evaluated by matching with these selected Offline tracks. 


\section{Efficiency versus Offline track $\mathrm{p}_{\mathrm{T}}$}

- Efficiencies with algorithms "out of the box"

- Good performance considering no additional tuning for data

- Excellent agreement with Monte Carlo

- EventFilter plateaus near $100 \%$
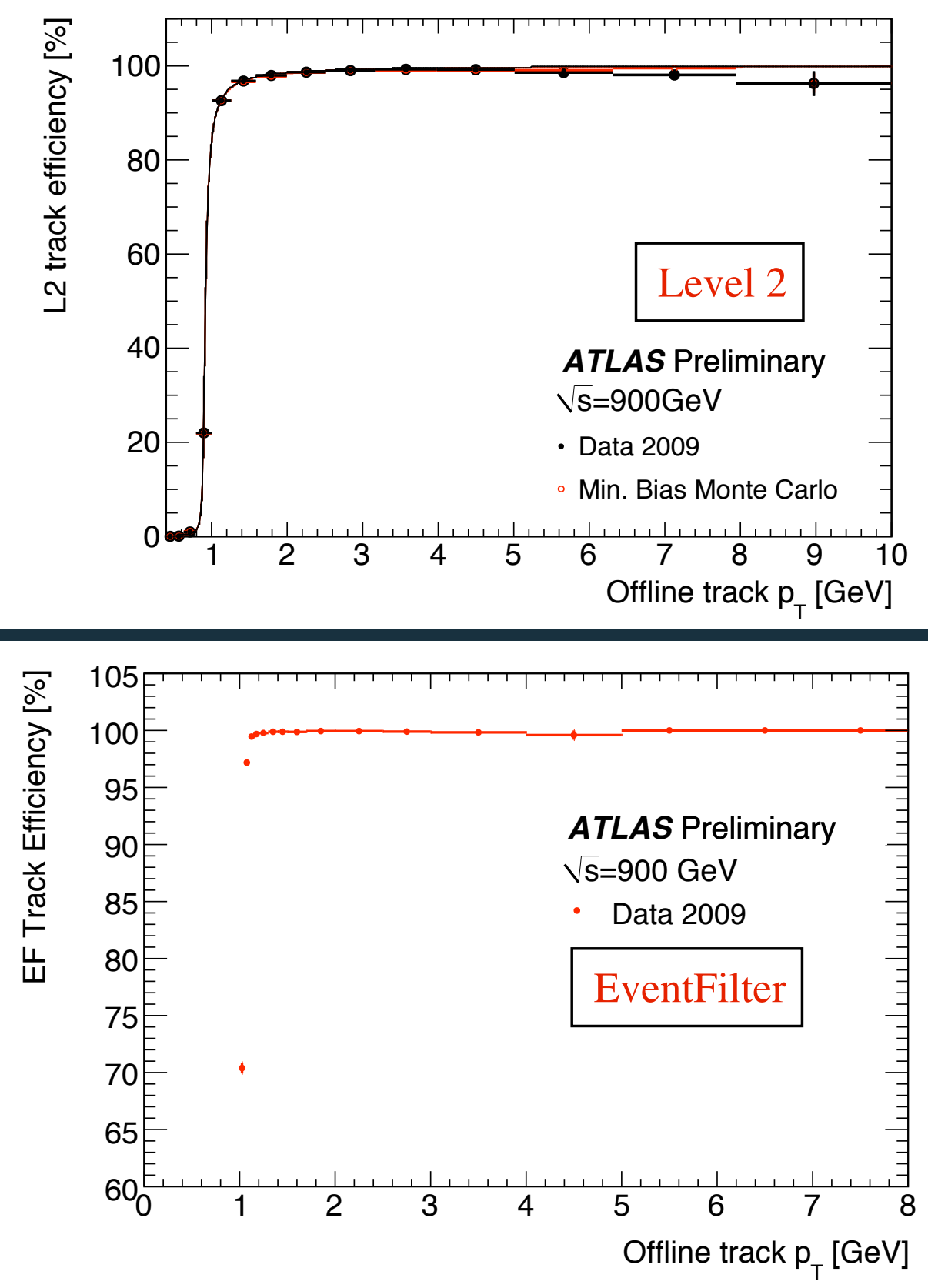


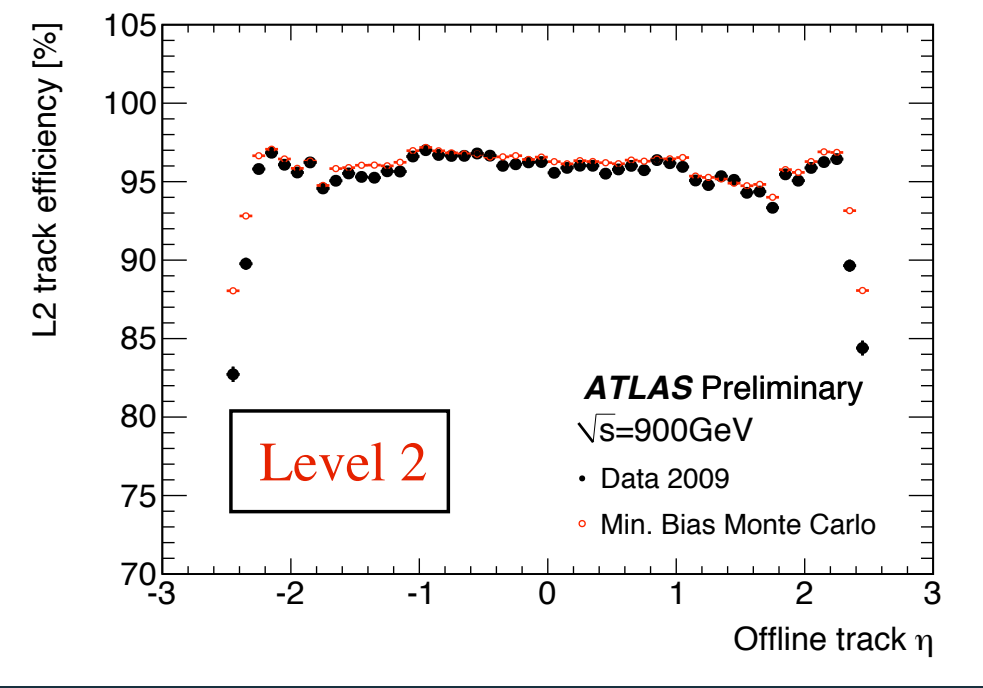

\section{Efficiency versus Offline track $\eta$}

- Well reproduced by the Monte Carlo, except at very large $|\eta|$

- Essentially flat efficiency versus $\eta$

- for EventFilter $\sim 100 \%$ for $\mathrm{p}_{\mathrm{T}}>1.2 \mathrm{GeV}$

- Higher efficiency for $7 \mathrm{TeV}$ due to harder track $p_{T}$ spectrum

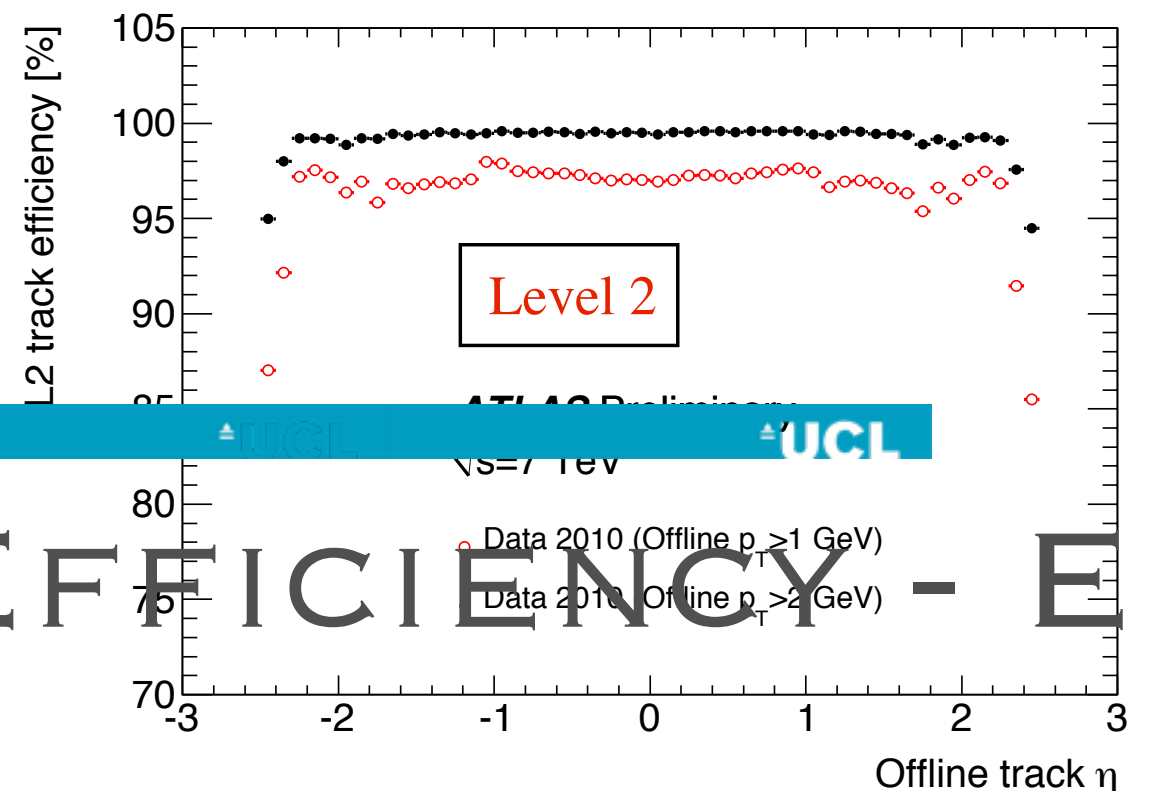

M.Sutton - The performance of the ATLAS Inner Detector Trigger 


\section{Efficiency vs time}
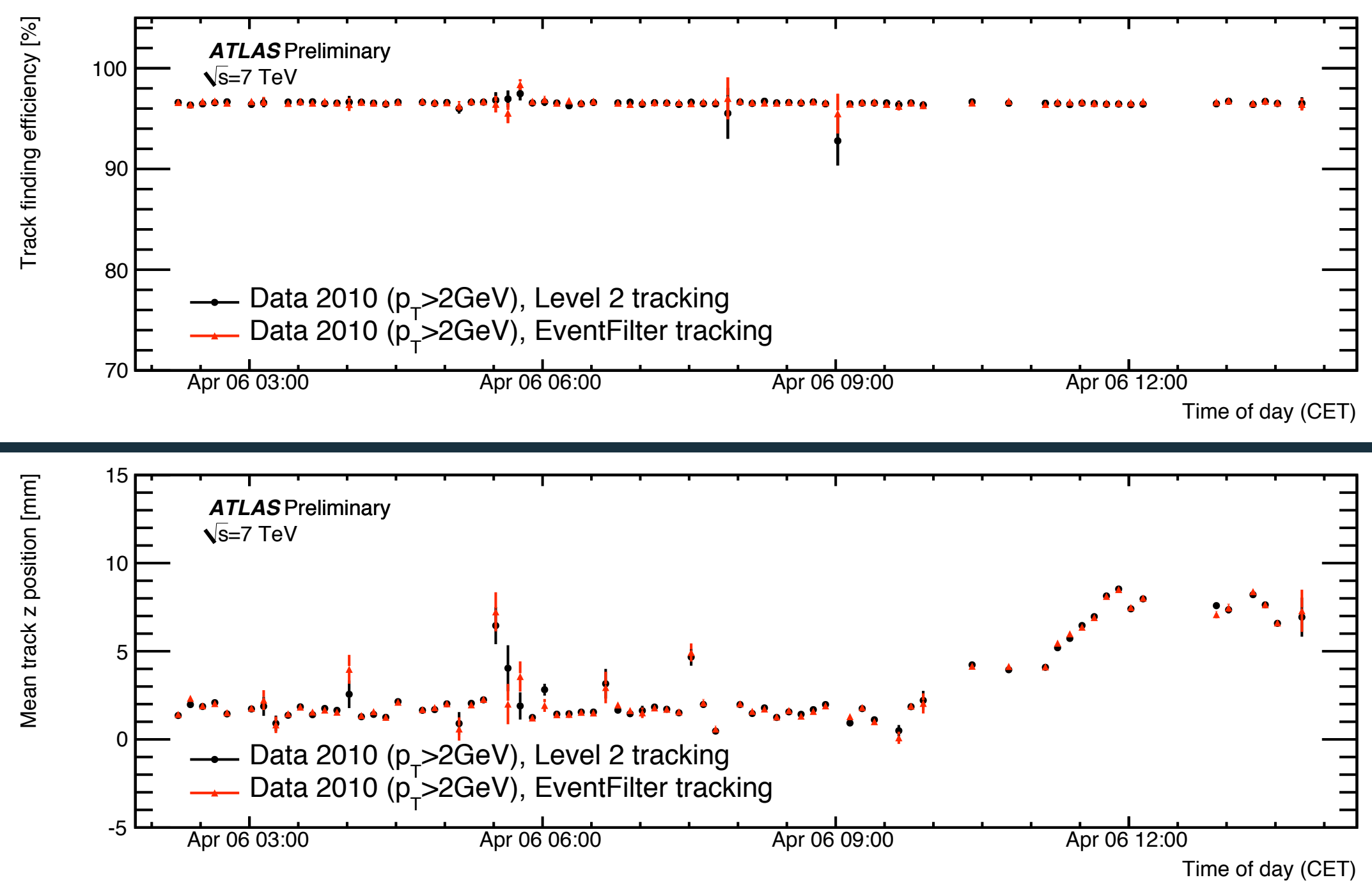

- Good stability of trigger over time with changing beam conditions 


\section{Transverse beam position}

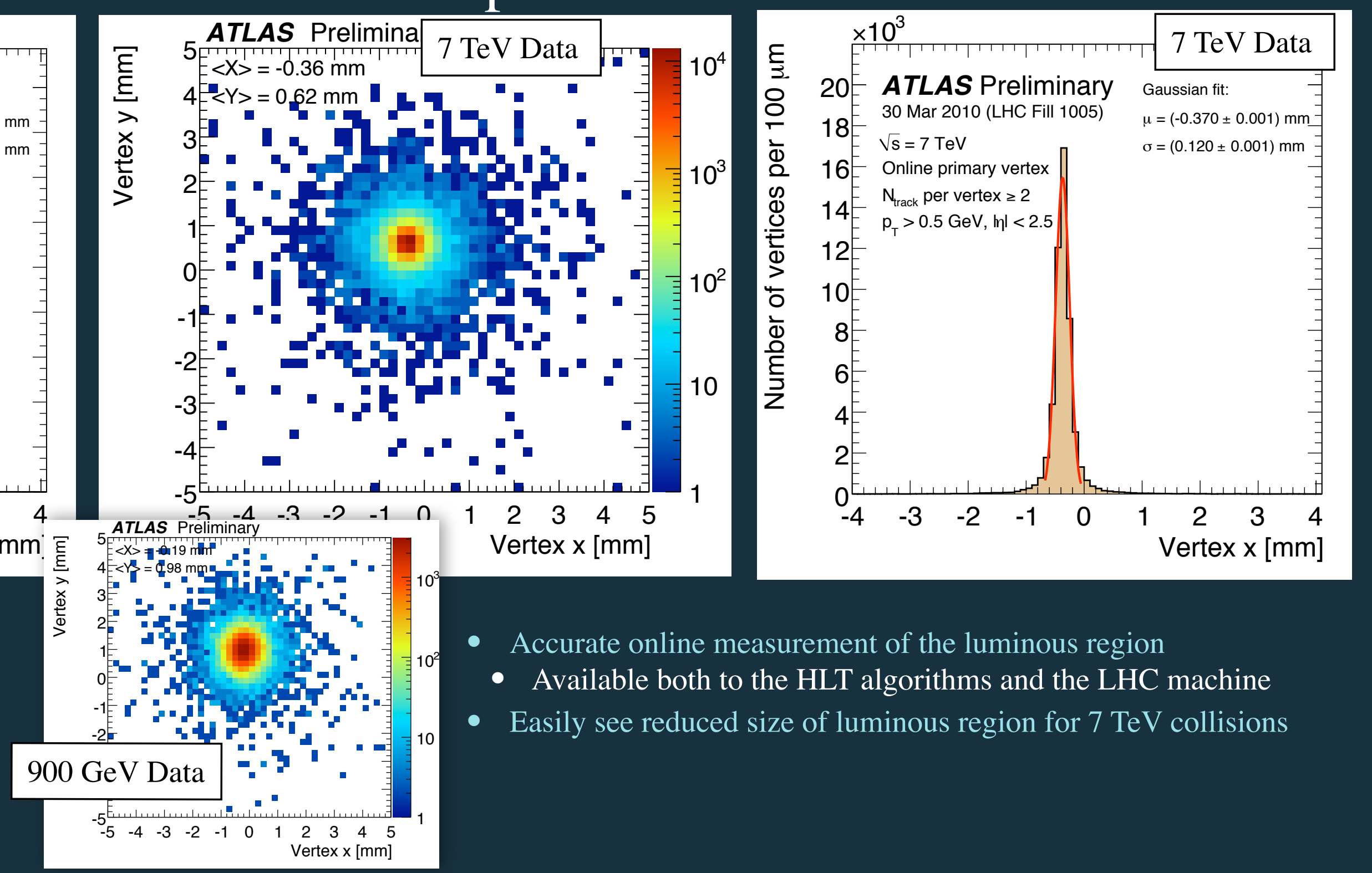




\section{Pixel hit multiplicities}

- Mean number of SCT and Pixel clusters on tracks versus offline track $\eta$

- Data well described by the Monte Carlo, including small number of dead Pixel modules

- Offline tracks reproduced by EventFilter reconstruction extremely well
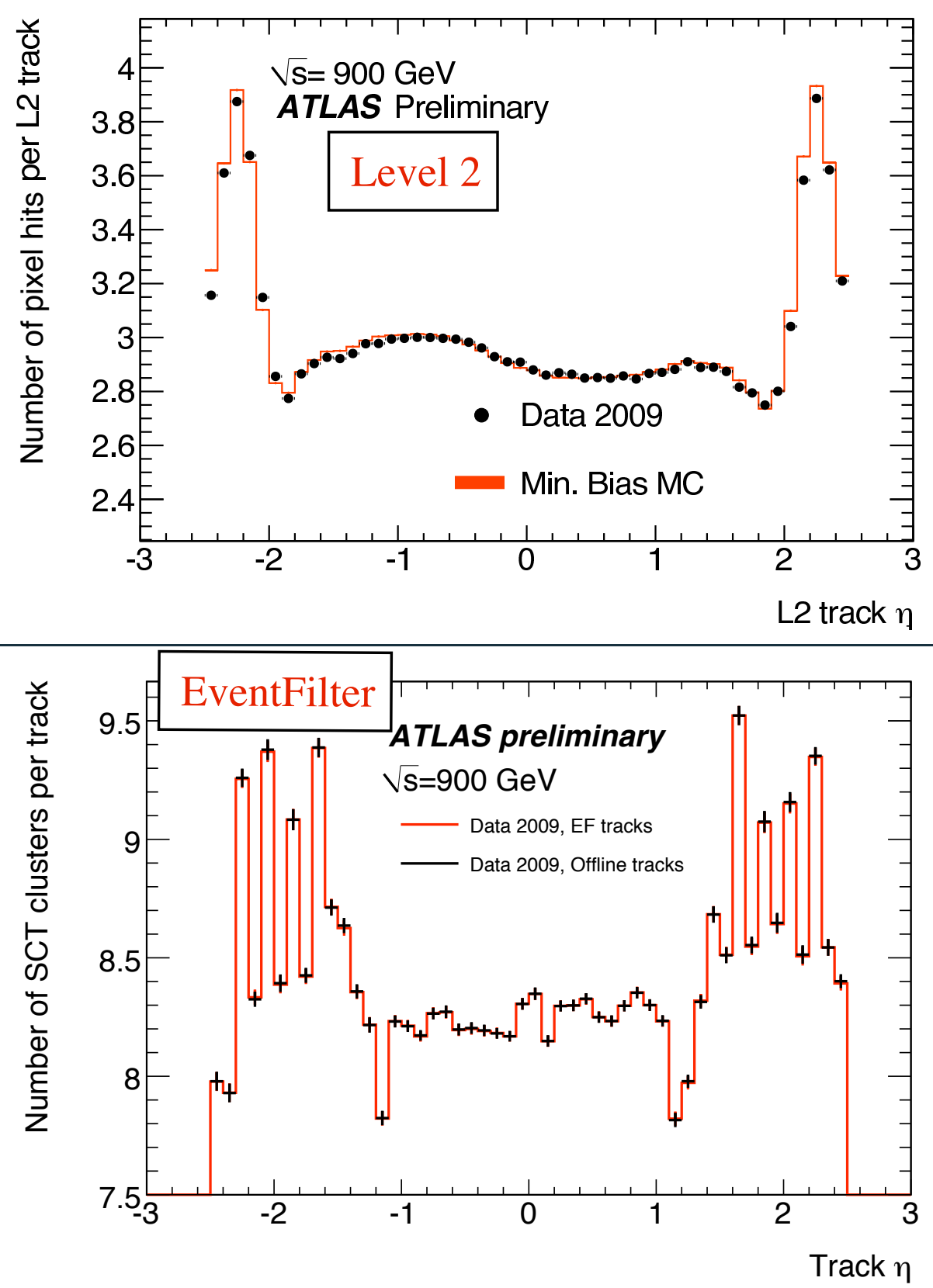
X11 Topical Seminar IPRD, Siena - 7-10 th June 2010

\section{Resolution with} respect to Offline

\section{track impact}

parameter
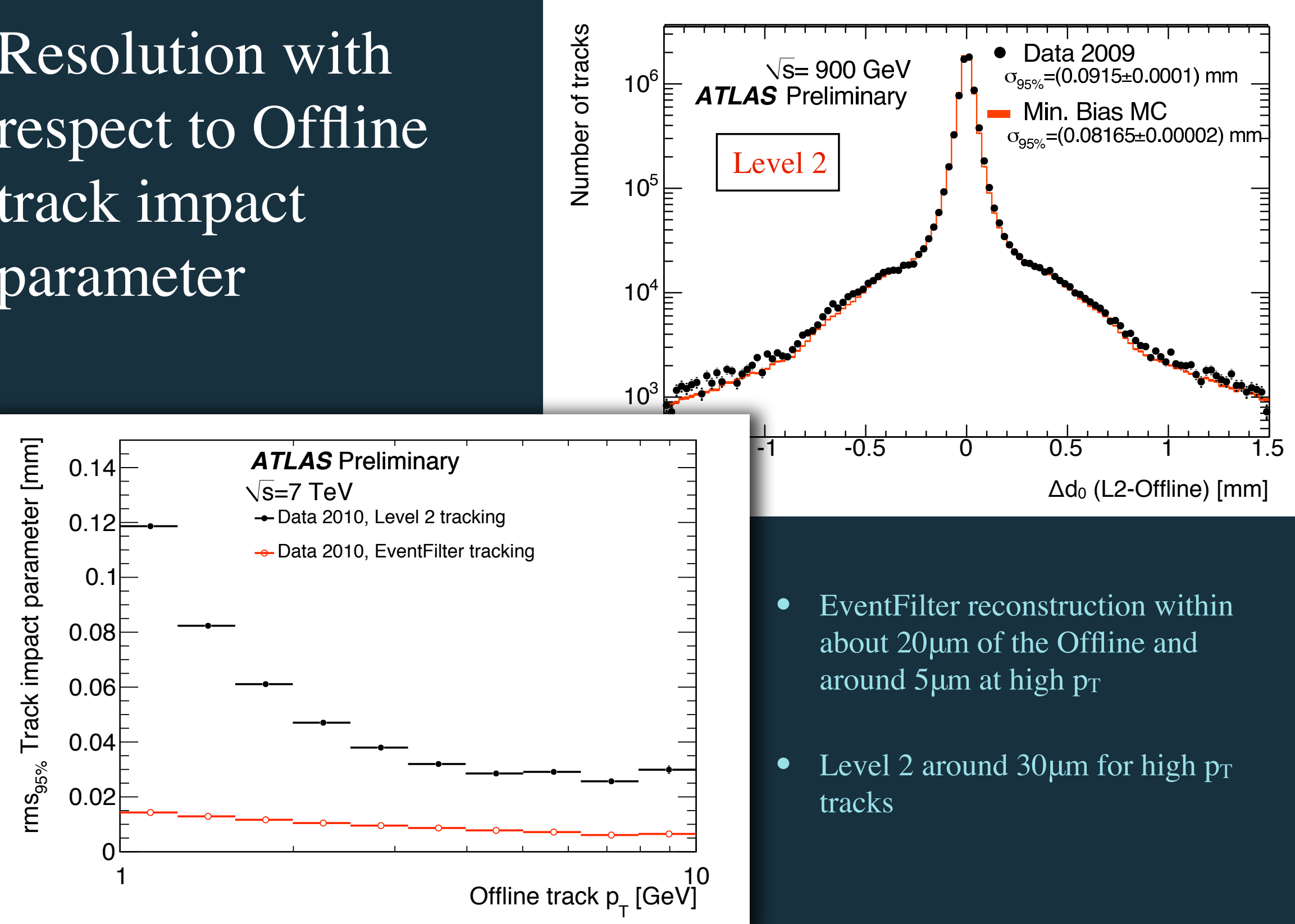

- EventFilter reconstruction within about $20 \mu \mathrm{m}$ of the Offline and around $5 \mu \mathrm{m}$ at high $\mathrm{p}_{\mathrm{T}}$

- Level 2 around $30 \mu \mathrm{m}$ for high $\mathrm{p}_{\mathrm{T}}$ tracks 


\section{Muon Trigger Efficiency}

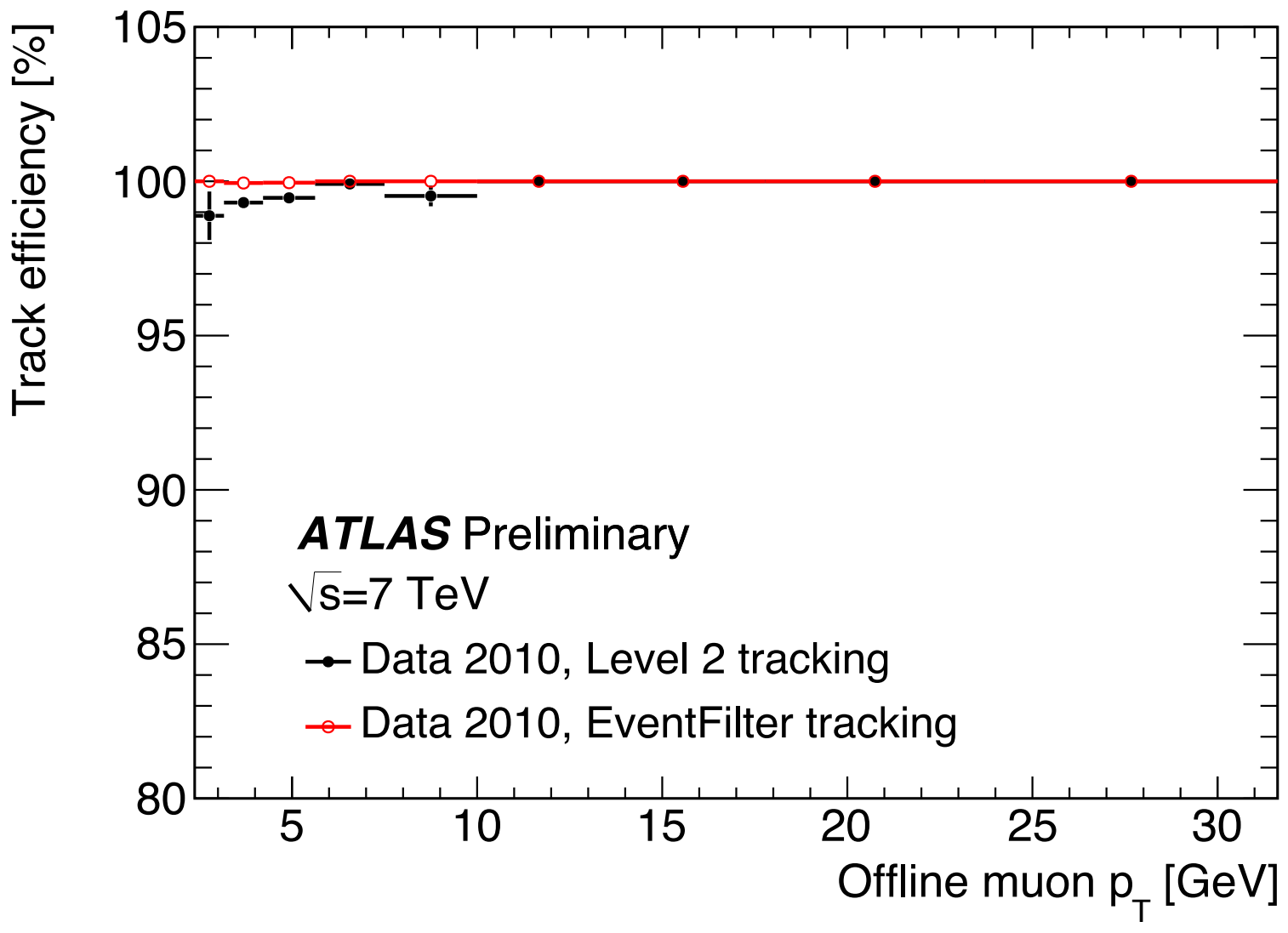

- Efficiency for reconstructing offline Muons with $\mathrm{p}_{\mathrm{T}}>4 \mathrm{GeV}$ with the Inner Detector Trigger in the Region of Interest identified by the L2 Muon algorithm

- Efficiency better than 99\% for both Level 2 and the EventFilter

M.Sutton - The performance of the ATLAS Inner Detector Trigger

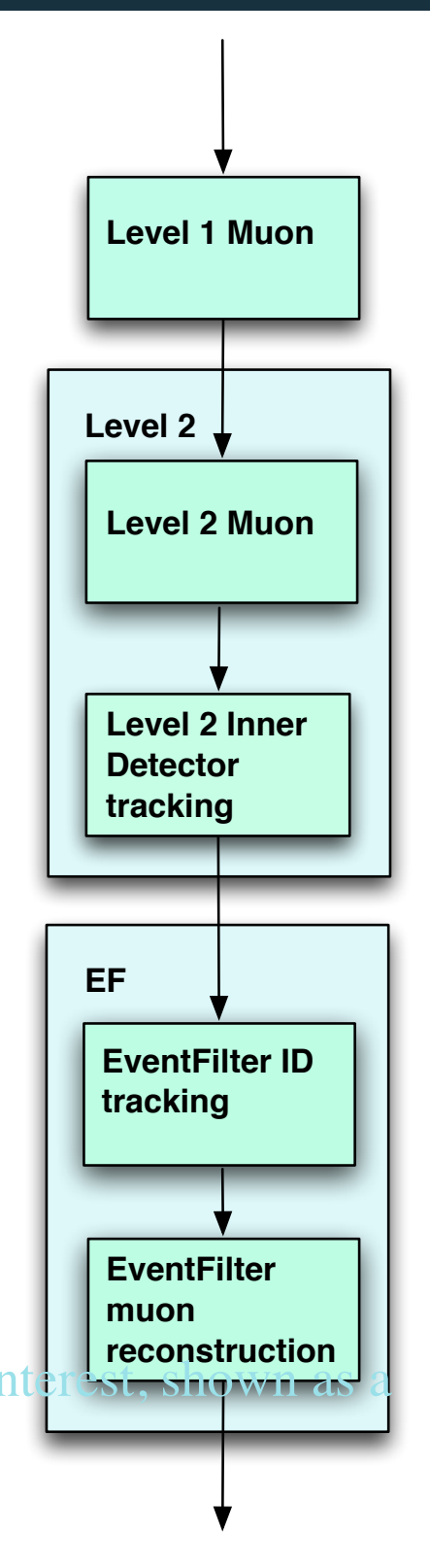




\section{Summary and Outlook}

- The LHC is routinely providing collisions at $7 \mathrm{TeV}$

- The HLT tracking is essential for the identification of physics objects with high transverse energy

- The ATLAS HLT tracking has been running successfully during data taking since December 2009, and also providing accurate online

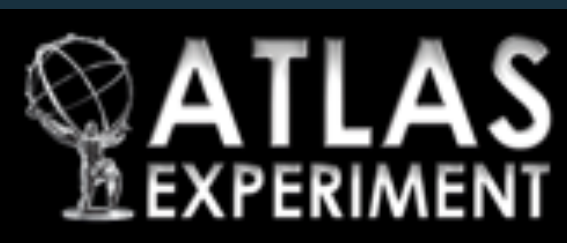

Run Number; 152221. Event Number; 383185

Date: 2010-04-01 00:31:22 CEST

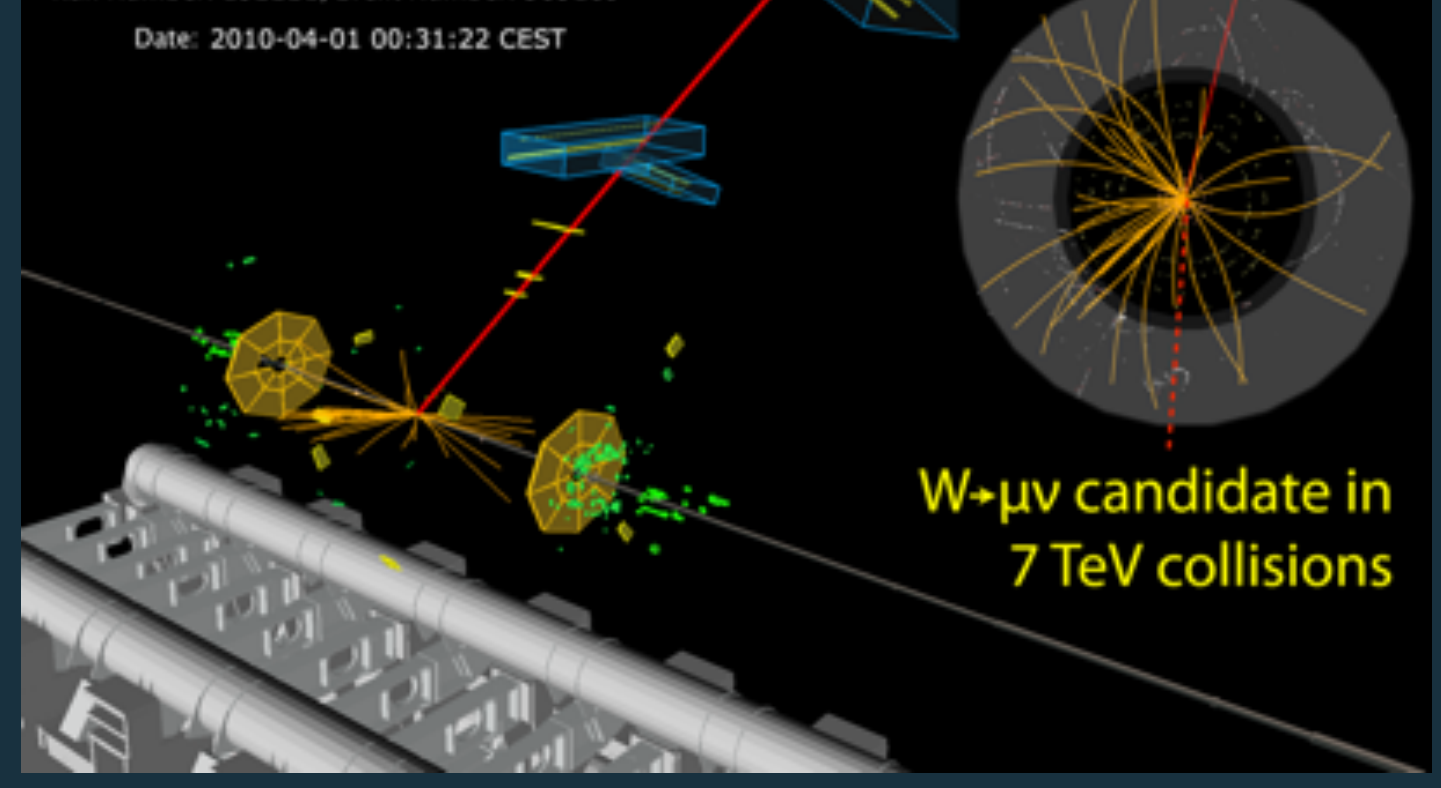
determination of the vertex

- Recently (since 23rd May) started running the HLT in rejection mode for the electron triggers.

- The HLT performance accurately reproduces that of the Offline algorithms and is remarkably well described by the Monte Carlo.

- The future will be an interesting time for us all. 